\title{
Nível de atividade física habitual e transtornos mentais comuns entre idosos residentes em áreas rurais
}

\author{
Level of routine physical activity and common mental disorders among elderly living in rural areas
}

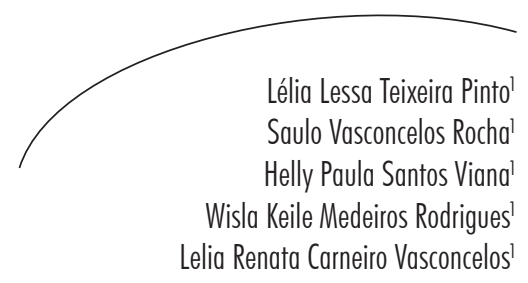

Resumo

Objetivo: Analisar a associação entre o nível de atividade física habitual e transtornos mentais comuns entre idosos. Métodos: Estudo transversal do tipo seccional com população de 95 idosos residentes na zona rural do município de Jequié-BA. Foram utilizados formulário com informações sociodemográficas, triagem para transtornos mentais comuns (TMC) através do Self-Reporting Questionnaire (SRQ-20) e o Questionário Internacional de Atividades Físicas (IPAQ). Resultados: A maioria dos idosos tinha entre 60 e 79 anos $(75,8 \%$ ) e era do sexo feminino (57,9\%). O percentual de não sedentários foi de $64,2 \%$, observando associação estatisticamente significante entre o nível de atividade física com a faixa etária, situação conjugal e escolaridade. A prevalência global de TMC foi de 47,4\%; apenas a renda teve associação estatisticamente significante. Ao avaliar a associação entre atividade física e TMC, não foi encontrada associação com níveis estatisticamente significante $(\mathrm{p}>0,05)$ Conclusão: Apesar das evidências da literatura sobre a contribuição da atividade física para a saúde mental, no presente estudo não se observou associação entre atividade física e TMC.

\section{Abstract}

Objective: To analyze the association between the level of habitual physical activity and common mental disorders among elderly. Methods: Cross-sectional study of 95 elderly people living in the rural area of Jequie city, state of Bahia, Brazil. A form with demographic information, screening for common mental disorders (CMD) with the Self-Reporting Questionnaire (SRQ-20) and the International Physical Activity Questionnaire (IPAQ) were used. Results: Most elderly were between 60 and 79 years old $(75.8 \%)$, female $(57.9 \%)$. The percentage of non-sedentary was $64.2 \%$, with statistically significant association between the level of physical activity and age, marital status and education. The overall prevalence of CMD was $47.4 \%$; only income had statistically significant association. To assess the association between physical activity and CMD, no association was found at statistically significant levels $(\mathrm{p}>0.05)$. Conclusion: Despite evidence from the literature on the contribution of physical activity for mental health, no association was observed between physical activity and CMD.

Núcleo de Estudos em Saúde da População. Universidade Estadual do Sudoeste da Bahia. Jequié, BA, Brasil.

Palavras-chave: Atividade Física. Saúde Mental. Idoso.

Key words: Physical Activity. Mental Health. Elderly. 


\section{INTRODUÇÃO}

O envelhecimento populacional, antes exclusivo dos países desenvolvidos, ocorre também nos países em desenvolvimento. ${ }^{1}$ No caso específico do Brasil, as alterações na dinâmica populacional são claras e significativas. Como consequência do crescimento da população idosa, a área do envelhecimento está se expandindo e sendo foco de pesquisa e atenção entre Estado, pesquisadores e sociedade. $^{2}$

Estudos têm sido conduzidos para melhor compreender as condições de vida e necessidades da população idosa. Contudo, a maioria desses estudos são direcionados para residentes em áreas urbanas, sendo escassas as ações direcionadas ao melhor entendimento da condição de saúde dos idosos residentes em zonas rurais. ${ }^{3-6}$

É notória a necessidade de agregar mais conhecimento sobre as condições de vida e saúde dos idosos residentes em áreas rurais. Essa população é mais vulnerável aos problemas decorrentes do avanço da idade, dentre eles os problemas de saúde mental. ${ }^{7}$

Dentre os problemas de saúde mental,
os transtornos mentais comuns (TMC) caracterizam-se por sintomas como insônia (privação do sono), fadiga (cansaço excessivo), irritabilidade (resposta excessiva a um estímulo), esquecimento (falha na retenção ou na evocação dos dados da memória), dificuldade de concentração (dificuldades de atenção) e queixas somáticas (diversos e variados sintomas físicos sem explicação) ${ }^{3,8}$ Os transtornos mentais comuns são mais prevalentes entre os indivíduos do sexo feminino, entre os idosos, divorciados ou viúvos, com menor grau de escolaridade e renda ${ }^{9,10}$ e entre os tabagistas. ${ }^{10}$

Na população idosa, com o aumento da idade, eleva-se a vulnerabilidade para os problemas de saúde mental.,11 Os idosos residentes em áreas rurais estão expostos a condições mais precárias de sobrevivência e maiores dificuldades estruturais para o acesso e utilização dos serviços de saúde, devido a fatores como a menor disponibilidade de serviços, escassez de transporte público, grande distância dos centros de saúde e baixa renda. ${ }^{12}$ Essas barreiras contribuem para uma percepção mais pessimista da qualidade de vida e a maior exposição às morbidades psíquicas.

Das terapias não medicamentosas disponíveis para prevenção e tratamento dos transtornos mentais, a atividade física apresenta-se como importante modalidade terapêutica, contribuindo para a melhora do bem-estar, autoestima, controle do estresse, da ansiedade e dos sintomas depressivos. ${ }^{3,13}$

Peluso \& Andrade ${ }^{14}$ apontam que a atividade física seria responsável por atuar diretamente nos fatores psicológicos (distração, autoeficácia e interação social) e nos fatores fisiológicos (aumento da transmissão sináptica das endorfinas, que supostamente funcionariam como as drogas antidepressivas) resultando, consequentemente, na melhora da ansiedade e do humor após a prática de atividade física.

Ainda são muito incipientes os estudos sobre atividade física e saúde mental na população idosa residente em área rural. Assim, este estudo teve como objetivo analisar a associação entre atividade física habitual e transtornos mentais comuns entre idosos residentes em áreas rurais.

\section{MÉTODO}

Trata-se de estudo transversal do tipo seccional, envolvendo uma população composta por indivíduos com idade igual ou superior a 60 anos residentes no distrito de Itajuru-BA e cadastrados na Unidade de Saúde da Família que cobre a zona rural do município de Jequié-BA.

O município de Jequié fica localizado na região sudoeste do estado baiano, possui área territorial de $3.035,42 \mathrm{Km}^{2}$, população estimada para o ano de 2010 de 151.820 habitantes. ${ }^{15}$ Nesse município, segundo dados do Instituto Brasileiro de Geografia e Estatística, ${ }^{16}$ a população de idosos é de 16.323, representando aproximadamente 
10,5\% da população total. O município conta com 28 Equipes de Saúde da Família (ESF), das quais duas são responsáveis pela cobertura dos idosos residentes na zona rural, sendo uma no distrito de Itajuru. ${ }^{17}$

Realizou-se levantamento nos registros da Unidade de Saúde da Família (USF) sobre o número de idosos cadastrados com idade de 60 anos ou mais. A USF contava com uma população de 103 idosos. Os critérios de inclusão utilizados foram: indivíduos com idade igual ou maior que 60 anos cadastrados pela ESF que atende ao distrito de Itajuru, zona rural do município de Jequié-BA. Foram excluídos todos os indivíduos com diagnóstico de demência ou qualquer outro tipo de alteração cognitiva e diagnóstico ou relato de surdez.

Após os critérios de exclusão adotados, a população final do estudo compreendeu 95 idosos (87\% do total de idosos cadastrados). Todos os avaliados assinaram um Termo de Consentimento Livre e Esclarecido, comprometendo-se a participar voluntariamente deste estudo.

Precedendo a coleta dos dados, foi conduzido treinamento com os entrevistadores. A coleta de dados foi realizada de outubro a dezembro de 2011 nas dependências da unidade de saúde. Os dados foram coletados por meio de formulário contendo informações sociodemográficas (idade, sexo, nível de escolaridade, situação conjugal e renda), comportamentais (etilismo, tabagismo), avaliação da atividade física habitual e transtornos mentais comuns.

Para avaliação da atividade física habitual, foi utilizado o Questionário Internacional de Atividades Físicas (IPAQ), versão adaptada para idosos. ${ }^{18}$ Esse instrumento mede o tempo semanal gasto na realização de atividade física de intensidade moderada a vigorosa em diferentes domínios (tarefas domésticas, trabalho, transporte e lazer); também mede o tempo despendido em atividades realizadas da posição sentada. Como ponto de corte, foram considerados indivíduos sedentários os idosos que, na soma das atividades físicas nos diferentes domínios, exercitaram menos de 150 minutos de atividades moderadas ou vigorosas por semana e os não sedentários os que praticaram acima desse valor. ${ }^{1}$

O Self Reporting Questionnaire (SRQ-20) foi o instrumento utilizado para a avaliação dos TMC. Validado por Gonçalves et al., ${ }^{19}$ tem como finalidade a identificação de casos psiquiátricos não psicóticos com a medida de pontuação mínima de 0 (nenhuma probabilidade) a 20 (extrema probabilidade). $\mathrm{Na}$ determinação de TMC, foi adotado o ponto de corte de cinco ou mais respostas positivas, procedimento adotado em outros estudos com a população idosa. ${ }^{20}$

$\mathrm{Na}$ análise dos dados, foram utilizados procedimentos da estatística descritiva e medidas de associação para variáveis categóricas (teste qui-quadrado). Para a associação entre atividade física habitual (variável de exposição) e os TMC (desfecho), foi utilizada a medida de associação razão de prevalência (RP). Utilizou-se a análise regressão linear simples para avaliar a correlação entre o tempo semanal de atividade física e o somatório de sintomas de TMC. Em todas as análises, o nível de significância adotado foi o valor de $\mathrm{p} \leq 0,05$.

O estudo foi aprovado pelo Comitê de Ética em Pesquisa da Universidade Estadual do Sudoeste da Bahia, sob parecer no 195/2010.

\section{RESULTADOS}

A idade média dos 95 idosos pesquisados foi de $73,54 \pm 9,43$ anos, sendo $57,9 \%$ do sexo feminino. Entre os entrevistados, a maioria era da faixa etária entre 60 e 79 anos (75,8\%), vivia sem companheiros $(52,6 \%)$ e possuía renda mensal de até um salário mínimo (96,2\%). Em relação às características comportamentais, identificou-se que a maioria não consumia bebidas alcoólicas e não tinha o hábito de fumar - 84,2\% e 80,5\%, respectivamente (tabela 1). 
Tabela 1. Características da população de idosos residentes em áreas rurais. Jequié-BA, 2011.

\begin{tabular}{|c|c|c|}
\hline Variável & $\mathrm{n}$ & $\%$ \\
\hline \multicolumn{3}{|l|}{ Sexo } \\
\hline Feminino & 55 & 57,9 \\
\hline Masculino & 40 & 42,1 \\
\hline \multicolumn{3}{|l|}{ Idade } \\
\hline 60-79 anos & 72 & 75,8 \\
\hline 80 ou mais & 23 & 24,2 \\
\hline \multicolumn{3}{|l|}{ Situação conjugal } \\
\hline Vive com companheiro & 45 & 47,4 \\
\hline Vive sem companheiro & 50 & 52,6 \\
\hline \multicolumn{3}{|l|}{ Escolaridade } \\
\hline Não alfabetizado & 62 & 65,3 \\
\hline Alfabetizado & 33 & 34,7 \\
\hline \multicolumn{3}{|l|}{ Renda } \\
\hline Até um salário mínimo & 76 & 96,2 \\
\hline Mais de um salário mínimo & 3 & 3,8 \\
\hline \multicolumn{3}{|l|}{ Tabagismo } \\
\hline Não & 70 & 80,5 \\
\hline $\operatorname{Sim}$ & 17 & 19,5 \\
\hline \multicolumn{3}{|l|}{ Etilismo } \\
\hline Não & 80 & 84,2 \\
\hline Sim & 15 & 15,8 \\
\hline
\end{tabular}

A frequência de idosos classificados como ativos fisicamente (ou seja, que praticavam 150 minutos ou mais de atividades físicas semanais de intensidade moderada e/ou intensa) foi de 64,2\%. Observou-se associação estatisticamente significante entre nível de atividade física com as variáveis "faixa etária" $(\mathrm{p}=0,004)$, "situação conjugal" ( $\mathrm{p}=0,020)$ e "escolaridade" $(\mathrm{p}=0,002)$, segundo a tabela 2 .
A prevalência global de TMC foi de 47,4\%. Observou-se associação estatisticamente significante apenas entre TMC e renda (tabela 3).

A prevalência de TMC foi menor entre os indivíduos não sedentários. Contudo, ao avaliar a associação entre atividade física e TMC, não foi encontrada associação com níveis estatisticamente significantes $(\mathrm{p}>0,05)$, como indica a tabela 4 . 
Tabela 2. Nível de atividade física segundo características da população. Jequié-BA, 2011.

\begin{tabular}{|c|c|c|}
\hline \multirow[t]{2}{*}{ Variável } & \multicolumn{2}{|c|}{ Não sedentários } \\
\hline & $\%$ & $\mathrm{p}$ valor \\
\hline \multicolumn{3}{|l|}{ Sexo } \\
\hline Masculino & 60,0 & 0,315 \\
\hline Feminino & 70,0 & \\
\hline \multicolumn{3}{|l|}{ Idade } \\
\hline 60-79 anos & 72,2 & 0,004 \\
\hline 80 ou mais & 39,1 & \\
\hline \multicolumn{3}{|l|}{ Situação Conjugal } \\
\hline Vive com companheiro & 75,6 & 0,020 \\
\hline Vive sem companheiro & 43,8 & \\
\hline \multicolumn{3}{|l|}{ Escolaridade } \\
\hline Não alfabetizado & 53,2 & 0,002 \\
\hline Alfabetizado & 84,8 & \\
\hline \multicolumn{3}{|l|}{ Renda } \\
\hline Até um salário mínimo & 59,2 & 0,15 \\
\hline Mais de um salário mínimo & 100 & \\
\hline \multicolumn{3}{|l|}{ Tabagismo } \\
\hline Não & 65,7 & 0,595 \\
\hline $\operatorname{Sim}$ & 58,8 & \\
\hline \multicolumn{3}{|l|}{ Etilismo } \\
\hline Não & 63,8 & 0,829 \\
\hline Sim & 63,7 & \\
\hline
\end{tabular}


Tabela 3. Transtornos mentais comuns (TMC) segundo características da população. Jequié-BA, 2011.

\begin{tabular}{|c|c|c|}
\hline \multirow[t]{2}{*}{ Variável } & \multicolumn{2}{|c|}{ Suspeito de TMC } \\
\hline & $\%$ & $\mathrm{p}$ valor \\
\hline \multicolumn{3}{|l|}{ Sexo } \\
\hline Masculino & 40,0 & 0,22 \\
\hline Feminino & 52,7 & \\
\hline \multicolumn{3}{|l|}{ Idade } \\
\hline 60-79 anos & 48,6 & 0,66 \\
\hline 80 ou mais & 43,5 & \\
\hline \multicolumn{3}{|l|}{ Situação Conjugal } \\
\hline Vive com companheiro & 42,2 & 0,341 \\
\hline Vive sem companheiro & 52,0 & \\
\hline \multicolumn{3}{|l|}{ Escolaridade } \\
\hline Não alfabetizado & 53,2 & 0,117 \\
\hline Alfabetizado & 36,4 & \\
\hline \multicolumn{3}{|l|}{ Renda } \\
\hline Até um salário mínimo & 46,1 & 0,05 \\
\hline Mais de um salário mínimo & 0,0 & \\
\hline \multicolumn{3}{|l|}{ Tabagismo } \\
\hline Não & 52,9 & 0,747 \\
\hline $\operatorname{Sim}$ & 48,6 & \\
\hline \multicolumn{3}{|l|}{ Etilismo } \\
\hline Não & 47,5 & 0,953 \\
\hline $\operatorname{Sim}$ & 46,7 & \\
\hline
\end{tabular}

Tabela 4. Associação entre atividade física e transtornos mentais comuns (TMC). Jequié-BA, 2011.

\begin{tabular}{lccc}
\hline & \multicolumn{3}{c}{ Suspeito de TMC } \\
\hline Atividade física & $\%$ & $\mathrm{RP}$ & $\mathrm{p}$ valor \\
Não sedentário & 46,2 & 1 & 0,208 \\
Sedentário & 48,8 & 1,29 & \\
\hline
\end{tabular}


Também não se identificou correlação entre tempo semanal gasto em atividades físicas e o somatório de sintomas de TMC $(\beta=5,51 \mathrm{p}=0,111)$, como demonstra a figura 1.

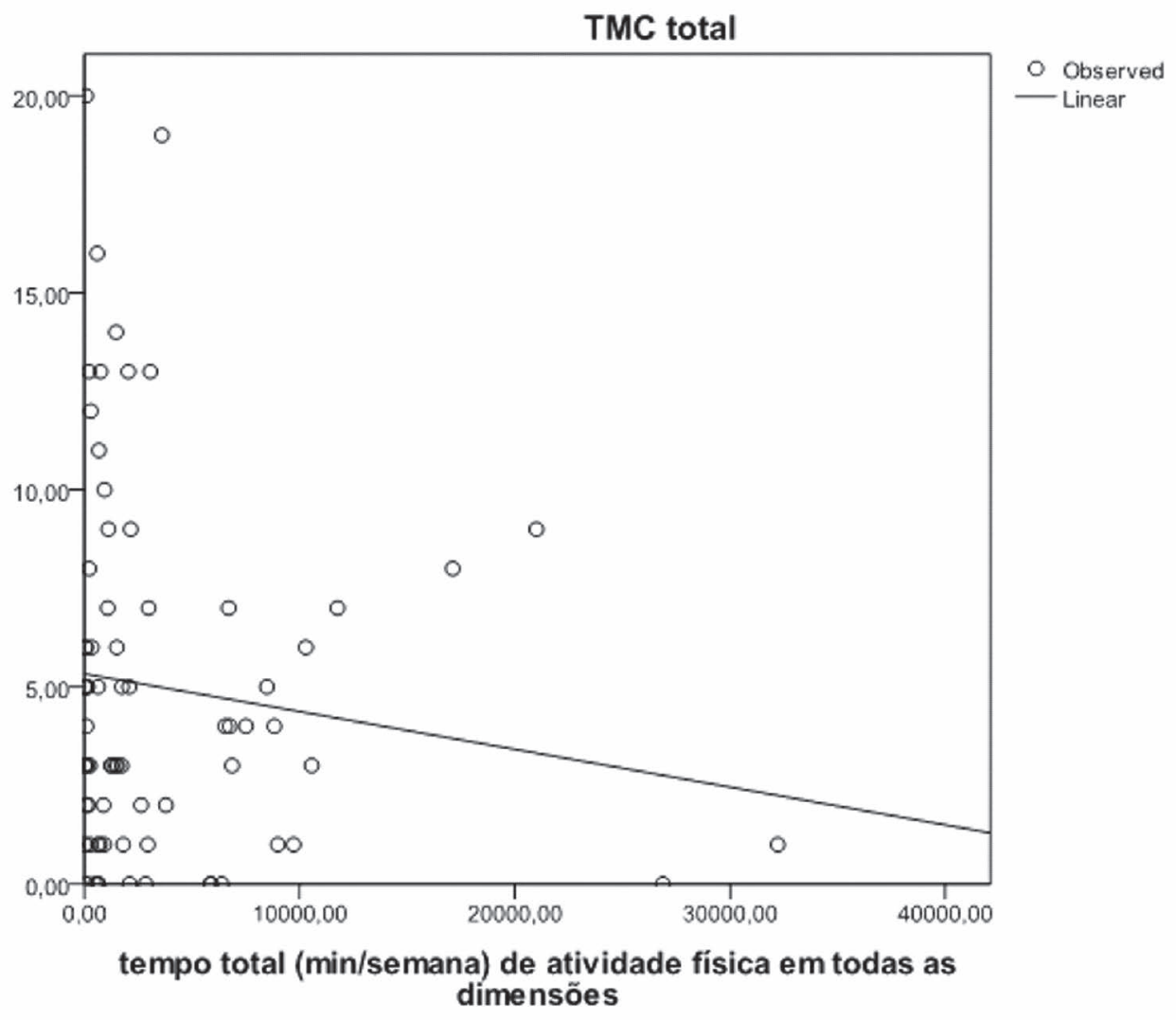

Figura 1. Tempo total de atividades físicas semanais e somatório de sintomas de transtornos mentais comuns (TMC). Jequié-BA, 2011.

\section{DISCUSSÃO}

$\mathrm{Na}$ presente investigação, a maioria dos entrevistados foi classificada como não sedentários para atividades físicas habituais, divergindo de estudos realizados com idosos de áreas rurais de Santa Catarina e Uberaba-MG, que identificaram predomínio de sedentarismo entre os idosos estudados. ${ }^{21,22}$ Madeira et al..$^{23}$ em estudo transversal com amostra de 12.402 indivíduos, sendo 6.624 idosos em cem municípios de 23 estados brasileiros, identificaram que $73,9 \%$ dos idosos estudados são inativos ( $<150$ minutos por semana). As condições de trabalho no âmbito rural, caracterizada pelo cultivo de lavouras mesmo após a aposentadoria, podem contribuir para a diminuição do sedentarismo entre essa população.

Idosos jovens que vivem com companheiro apresentaram-se como mais ativos, conforme encontrado em outros estudos. ${ }^{24,25}$ A idade é fator limitante para a prática de atividade física, pois com o avançar dos anos, as condições fisiológicas impedem a realização de diversos exercícios que contribuem para uma vida mais saudável. ${ }^{25}$ Observou-se que as mulheres foram consideradas menos sedentárias que os homens, corroborando achados de outros estudos. ${ }^{1,26} \mathrm{~A}$ atividade física também esteve associada com o nível de escolaridade, partindo-se da premissa que as atividades laborais realizadas no campo 
exigem maior força física e menor relação com a escolaridade dos trabalhadores. ${ }^{24}$

Com relação à condição de saúde mental, Costa \& Ludermir ${ }^{9}$ destacam que a prevalência de TMC em áreas rurais está próxima da apresentada em áreas urbanas, confirmando os achados do presente estudo. Quando analisados os fatores associados aos TMC, verificou-se que as mulheres e os idosos jovens (60-79 anos) estão mais expostos aos TMC, confirmando os resultados encontrados em outros estudos. ${ }^{1,3,5,9,27}$

A associação entre a renda e o transtorno mental comum pode estar relacionada com as condições inadequadas de vida, como o estresse e insegurança devido à renda insuficiente, desencadeando TMC. ${ }^{3}$

No presente estudo, a atividade física não esteve associada aos TMC, divergindo dos estudos realizados por Benedetti et al., ${ }^{1}$ Minghelli et al. ${ }^{28}$ e Rocha et al. ${ }^{27}$ que verificaram associação inversa entre a prática de atividade física e a prevalência de transtornos mentais, demonstrando que a atividade física regular pode ser fator de proteção para TMC.

Dentre os participantes do programa, a prática regular de atividades físicas no tempo livre proporciona redução nos sintomas dos TMC, uma vez que favorece a interação social e proporciona melhoria nos sintomas depressivos, ansiedade, tolerância ao estresse e aumento da autoestima e da sensação de bem-estar. Quando praticadas em grupo elevam a autoestima, contribuindo para a construção das relações psicossociais e para o reequilíbrio emocional. ${ }^{4}$

Estudo conduzido no norte da Espanha com 436 idosos concluiu que a prática de atividade física esteve relacionada tanto com a melhora da capacidade física, quanto com a diminuição dos sintomas depressivos entre os idosos estudados. ${ }^{29}$

Éimportante ressaltara relevância da assistência aos indivíduos idosos quanto aos transtornos mentais. Estes podem ter início de forma lenta e insidiosa, ${ }^{30}$ variando o tempo de progressão da doença; ${ }^{31}$ daí a importância do rastreamento, avaliação e acompanhamento desses indivíduos para a identificação desse transtorno.

Christofoletti et al. ${ }^{32}$ realizaram estudo de revisão que indicava que intervenções não farmacológicas associadas à prática de atividades físicas são importantes instrumentos para a promoção de benefícios psicossociais, status afetivo, estresse do cuidador e saúde física.

Dentre as limitações deste estudo, pode-se destacar o desenho da pesquisa, visto que um estudo de corte transversal limita a avalição da causa e efeito das variáveis. Portanto, não se pode avaliar se a atividade física habitual influenciou na presença de TMC. Além disso, pode ter ocorrido o viés de informação, principalmente pelo uso do IPAQ, devido à dificuldade de entendimento dos entrevistados e a descontextualização das atividades físicas descritas no instrumento com as atividades realizadas no ambiente rural. Em contrapartida, este estudo foi conduzido com uma população pouco estudada no Brasil, podendo servir como insumo para elaboração de ações direcionadas para esse segmento.

\section{CONCLUSÃO}

A prática de atividade física não se associou a redução dos transtornos mentais comuns entre idosos residentes em áreas rurais. Contudo, a associação, apesar de não ser estatisticamente significante, demonstra um sensível aumento dos transtornos mentais comuns entre os indivíduos sedentários.

Recomenda-se a realização de novos estudos com populações maiores e levantamentos longitudinais, no sentido de esclarecer melhor a associação entre essas variáveis na população idosa residente em área rural.

Sugere-se, ainda, que fatores associados aos hábitos de vida na zona rural sejam aspectos que possam ser incorporados em novos estudos, tendo em vista as particularidades da população rural, principalmente os idosos, os quais muito provavelmente são mais envolvidos com atividades habituais menos estressantes. 


\section{REFERÊNCIAS}

1. Benedetti TRB, Borges LJ, Petroski EL, Gonçalves LHT. Atividade física e estado de saúde mental de idosos. Rev Saúde Pública 2008; 42(2):302-7.

2. Garrido R, Menezes PR. O Brasil está envelhecendo: boas e más notícias por uma perspectiva epidemiológica. Rev Bras Psiquiatr 2002;24(Supl 1):3-6.

3. Rocha SV, De Almeida MMG, De Araujo TM, Virtuoso JS Junior. Prevalência de transtornos mentais comuns entre residentes em áreas urbanas de Feira de Santana, Bahia. Rev Bras Epidemiol 2010;13(4):630-40.

4. Rocha SV, De Araujo TM, De Almeida MMG, Virtuoso JS Junior. Prática de atividade física no lazer e transtornos mentais comuns entre residentes de um município do nordeste do Brasil. Rev Bras Epidemiol 2012;15(4):871-83.

5. Adamoli AN, Azevedo MR. Padrões de atividade física de pessoas com transtornos mentais e de comportamento. Ciênc Saúde Coletiva 2009;14(1):243-251.

6. Cheik NC, Reis IT, Heredia RAG, Ventura ML, Tufik $\mathrm{S}$, Antunes HK, et al. Effects of the physical exercise and physical activity on the depression and anxiety in elderly. R Bras Ciênc Mov 2003;11(3):45-52.

7. Paz AA, Santos BRL, Eidt OR. Vulnerabilidade e envelhecimento no contexto da saúde. Acta Paul Enferm 2006;19(3):338-42.

8. Ludermir AB, Melo Filho DA. Condições de vida e estrutura ocupacional associadas a transtornos mentais comuns. Rev Saúde Pública 2002;36(2):213-21.

9. Costa AG, Ludermir AB. Transtornos mentais comuns e apoio social: estudo em comunidade rural da Zona da Mata de Pernambuco, Brasil. Cad Saúde Pública 2005;21(1)73-9.

10. Li-Jung C, Stevinson C, Po-Wen K, Yu-Kai C, Da-Chen C. Relationships of leisure-time and non-leisure-time physical activity with depressive symptoms: a population-based study of Taiwanese older adults. Int J Behav Nutr Phys Act 2012;9:1-10.

11. Stella F, Gobbi S, Corazza DI, Costa JLR. Depressão no idoso: diagnóstico, tratamento e benefícios da atividade física. Motriz 2002;8(3):91-8.

12. Travassos C, Viacava F. Acesso e uso de serviços de saúde em idosos residentes em áreas rurais, Brasil, 1998 e 2003. Cad Saúde Pública 2007;23(10):2490-502.

13. Akke KVDB, Miranda GHL, Wensing M. Effectiveness of physical activity interventions for older adults: a review. Am J Prev Med 2002;22(2):120-33.
14. Peluso MAM, Andrade LHSG. Physical activity and mental health: the association between exercise and mood. Clinics 2005;60(1):61-70.

15. Instituto Brasileiro de Geografia e Estatística. Censo demográfico. Características da população e dos domicílios: resultados do universo. Rio de Janeiro: IBGE; 2010.

16. Instituto Brasileiro de Geografia e Estatística. Censo demográfico. Características da população e dos domicílios: resultados do universo. Rio de Janeiro: IBGE; 2009.

17. Organização Mundial de Saúde. Relatório sobre saúde no mundo: saúde mental: nova concepção, nova esperança. Geneve: OMS; 2001.

18. Mazo GZ, Benedetti TRB. Adaptação do questionário internacional de atividade física para idosos. Rev Bras Cineantropom Desempenho Hum 2010,12(6):480-84.

19. Gonçalves DM, Stein AT, Kapczinski F. Avaliação de desempenho do Self-Reporting Questionnaire como instrumento de rastreamento psiquiátrico: um estudo comparativo com o Structured Clinical Interview for DSM-IV-TR. Cad Saúde Pública 2008;24(2):380-90.

20. Scazufca M, Menezes PR, Vallada H, Araya R. Validity of the Self-Reporting Questionnaire-20 in epidemiological studies with older adults. Soc Psychiatr Psychiatr Epidemiol 2009;44(3):247-54.

21. Santos KA, Koszuoski R, Dias-da-Costa JS, Pattussi MP. Fatores associados com a incapacidade funcional em idosos do Município de Guatambu, Santa Catarina, Brasil. Cad Saúde Pública 2007;23(11):2781-88.

22. Tavares DMS, Arduini AB, Dias FA, Ferreira PCS, De Oliveira EA. Perfil sociodemográfico, capacidade funcional e qualidade de vida de homens idosos residentes na zona rural. Rev Enferm Atenção Saúde 2012;1(1):16-29.

23. Madeira MC, Siqueira FCV, Facchini LA, Silveira DS, Tomasi E, Thumé E, et al. Atividade física no deslocamento em adultos e idosos do Brasil: prevalências e fatores associados. Cad Saúde Pública 2013;29(1):165-74.

24. Bicalho PG, Hallal PC, Gazzinelli A, Knuth AG, Velásquez-Meléndez G. Atividade física e fatores associados em adultos de área rural em Minas Gerais, Brasil. Rev Saúde Pública 2010;44(5):884-93.

25. Cassou ACN, Fermino RC, Santos MS, RodriguezAñez C, Reis RS. Barreiras para a atividade física em idosos: uma análise por grupos focais. Rev Edic Fís 2008;19(3):353-60. 
26. Adamoli AN, Azevedo M R. Padrões de atividade física de pessoas com transtornos mentais e de comportamento. Ciênc Saúde Coletiva 2009;14(1):243-51.

27. Rocha SV, Rocha SV, De Almeida MMG, De Araujo TM, Virtuoso JS Junior. Atividade física no lazer e transtornos mentais comuns entre idosos residentes em um município do nordeste do Brasil. J Bras Psiquiatr 2011;60(2):80-5.

28. Minghelli B, Tomé B, Nunes C, Neves A, Simões C. Comparação dos níveis de ansiedade e depressão entre idosos ativos e sedentários. Rev Psiquiatr Clín 2013;40(2):71-6.

29. Salguero A, García-Martínez R, Molinero O, Márquez S. Physical activity, quality of life and symptoms of depression in community-dwelling and institutionalized older adults. Arch Gerontol Geriatr 2011;53(2):152-7.

30. Tavares A, Organizador. Compêndio de neuropsiquiatria geriátrica. Rio de Janeiro: Guanabara Koogan; 2005. p. 217-34.

31. Machado JCB. Doença de Alzheimer. In: Freitas EV, Py L, Neri AL, Cançado FAX, Gorzoni ML, Rocha SM, editores. Tratado de Geriatria e Gerontologia. Rio de Janeiro: Editora Guanabara Koogan; 2006. p. 260-80.

32. Christofoletti G, Oliani MM, Bucken-Gobbi LT, Gobbi S, Beinotti F, Stella F. Physical activity attenuates neuropsychiatric disturbances and caregiver burden in patients with dementia. Clinics 2011;66(4):613-8. 